\title{
ROCK as a Therapeutic Target of Diabetic Retinopathy
}

\author{
Ryoichi Arita, Yasuaki Hata, and Tatsuro Ishibashi \\ Department of Ophthalmology, Graduate School of Medical Sciences, Kyushu University, 3-1-1 Maidashi, Higashi-Ku, \\ Fukuoka 812-8582, Japan \\ Correspondence should be addressed to Yasuaki Hata, hatachan@med.kyushu-u.ac.jp
}

Received 2 December 2009; Accepted 9 April 2010

Academic Editor: Kavita Hegde

Copyright (c) 2010 Ryoichi Arita et al. This is an open access article distributed under the Creative Commons Attribution License, which permits unrestricted use, distribution, and reproduction in any medium, provided the original work is properly cited.

\begin{abstract}
The increasing global prevalence of diabetes is a critical problem for public health. In particular, diabetic retinopathy, a prevalent ocular complication of diabetes mellitus, causes severe vision loss in working population. A better understanding of the pathogenesis and the development of new pharmacologic treatments are needed. This paper describes the relevance between Rho/ROCK pathway and the pathogenesis of diabetic retinopathy from its early to late stages. Moreover, the therapeutic potential of ROCK inhibitor in the total management of diabetic retinopathy is discussed.
\end{abstract}

\section{Introduction}

The growing prevalence of diabetic retinopathy (DR), the common ocular complication of diabetes mellitus, is a critical problem for global public health [1, 2]. Early nonproliferative stages of DR are characterized by blot hemorrhages and vascular abnormality such as retinal vascular microaneurysms or hyperpermeability which could cause diabetic macular edema (DME). Proliferative diabetic retinopathy (PDR), later stage of the diseased state, causes neovascularization, vitreous hemorrhages, preretinal fibrovascular proliferation, and tractional retinal detachment. While visual acuity is not always affected in early stages, progression of the disease leads to severe vision loss.

Panretinal photocoagulation (PRP) and vitreoretinal surgery remain the primary therapeutic strategies for progressed DR. However, PRP is destructive to the retina and accompanied by adverse effects such as decreased visual acuity, increased risk of macular edema, and pain [3, 4]. Moreover, recent advances in vitreous surgery cannot always achieve a satisfying visual acuity [5]. Now it is at a point where new alternative and adjunctive agents from the earlier stages are urgently required because of overwhelming patient's physical and economic burdens of these treatments.

The various clinical findings in earlier DR stages are related to endothelial damage secondary to increased leukocyte adhesion mediated through adhesion molecules, intercellular adhesion molecule-1 (ICAM-1), and leukocyte $\beta_{2}$-integrins (CD18/CD11a and CD18/CD11b) [6-8]. In addition, the critical mechanism of this leukocyte-induced endothelial damage is the interaction of endothelial Fas with Fas ligand, expressed on adherent leukocytes in diabetic rats [9] and DR patients [10].

Recent accumulating evidences indicate that vascular endothelial growth factor (VEGF) plays a critical role in pathogenesis of both DME and neovascularization in PDR $[11,12]$. Clinical studies with anti-VEGF antibodies are potentially useful strategy and improve outcome for treating DR [13]. However, the adaptation is only for progressed states and there is a possibility of systemic adverse complications such as hypertension, cerebrovascular accidents, and myocardial infarcts by anti-VEGF therapy [14].

Meanwhile, neovascularization and proliferative vitreoretinopathy (PVR) are hallmark of the later DR stages. VEGF-induced endothelial migration and proliferation is essential process for angiogenesis. ERK1/2 signaling promotes the proliferative activities of endothelial cells in angiogenic processes $[15,16]$. Moreover, endothelial migration is mediated by Rho-kinase (ROCK) pathway which activates remodeling of endothelial F-actin cytoskeleton [17].

Our recent findings indicated that hyalocytes, a known resident macrophage in the cortical vitreous under physiological conditions, appeared to be involved in the pathogenesis of PVR associated with a cicatricial contraction of 


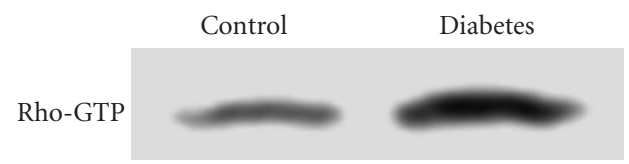

(d)

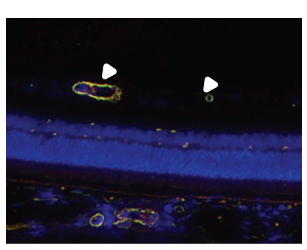

(a)

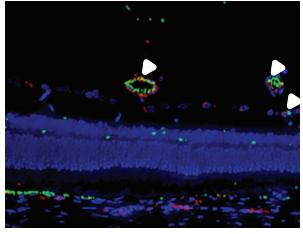

(b)

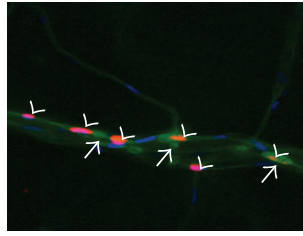

Diabetes + vehicle

(g)

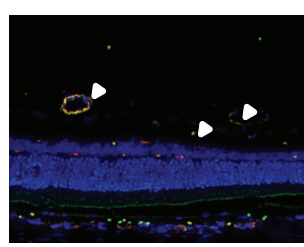

(c)

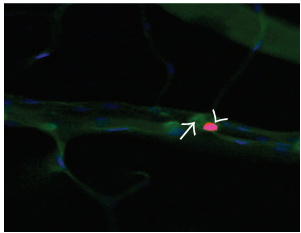

Diabetes + fasudil

(h)

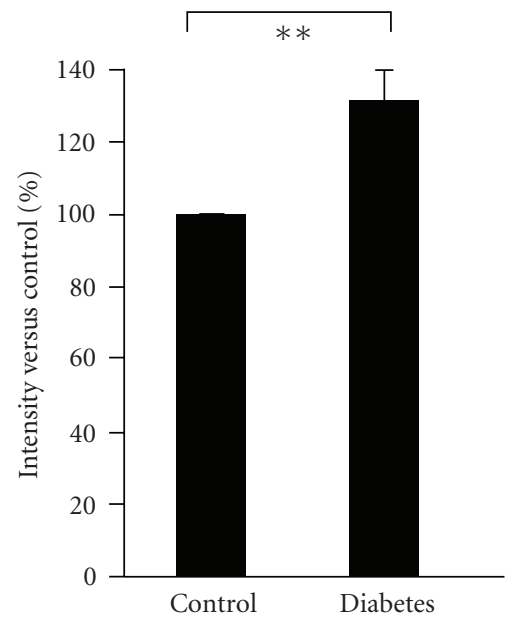

(e)

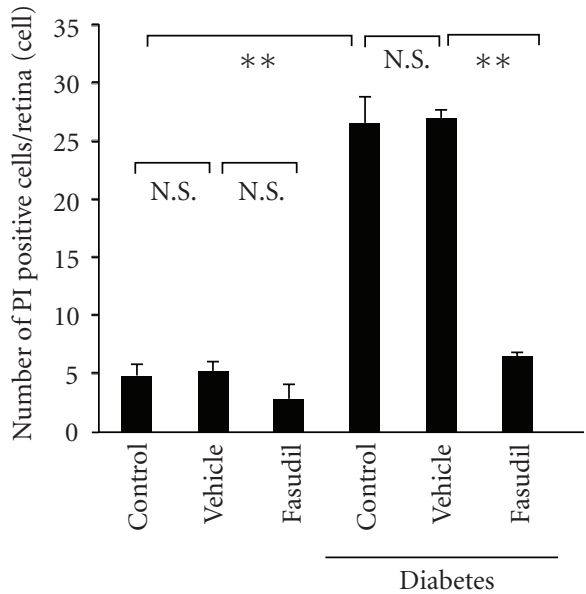

(i)

FIgURE 1: Rho/ROCK activation in retinal vessels. In immunohistochemical analysis, RhoA (a), ROCK1 (b) and ROCK2 (c) were detected in retinal vessels. Yellow (white arrowhead) indicates double-stained vasculature (magnification: $\times 400)$. ( $\mathrm{d}$ and e) The levels of Rho-GTP were significantly higher in streptozotocin induced-diabetic rat bretinas, compared with those in nondiabetic control, detected by Rho pull-down assay. Average signal intensities are quantified and expressed as percentage of the ratio of control (** $P<.01, n=5$ each). Prevention of leukocyte-induced retinal endothelial damage by fasudil. ( $\mathrm{f}-\mathrm{i}$ ) In vivo visualization of adhering leukocytes (green, concanavalin A lectin) and injured endothelial cells (red, propidium iodide (PI)) and endothelial nuclei (blue, DAPI) in rat retinas. PI positive cells (white arrowhead) widely coincided with adherent leukocytes (white arrow). The number of PI positive cells per retina was significantly higher in the diabetic animals, compared with the nondiabetic controls. Fasudil caused a significant reduction in the number of PI positive cells in the retinas of the diabetic animals, compared with the vehicle-treated controls ( ${ }^{*} P<.01$, N.S., not significant, $n=5$ each).

proliferative membranes in PDR [18-20]. The expression of $\alpha$-smooth muscle actin ( $\alpha$-SMA) and phosphorylation of myosin light chain (MLC) in hyalocytes, which is associated with stress-fiber formation and contractile rings, are facilitating cell contraction $[21,22]$. We demonstrated that vitreous from patients with PDR significantly promoted expression of $\alpha$-SMA and phosphorylation of MLC, and enhanced contraction of hyalocyte-containing collagen gels, compared with vitreous from patients with nondiabetic controls [23].

In this paper, we first place the Rho/ROCK pathway plays a critical role in diabetic retinal microvasculopathy, neovascularization, and tractional retinal detachment associated with a cicatricial contraction of proliferative membranes. We then describe our current knowledge about ROCK inhibition 

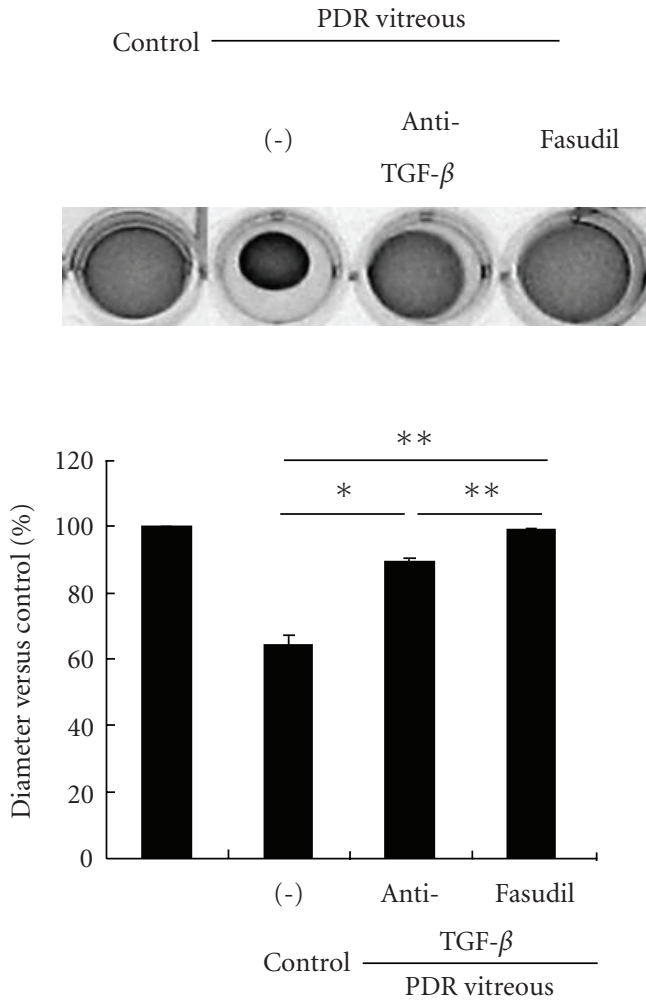

(a)
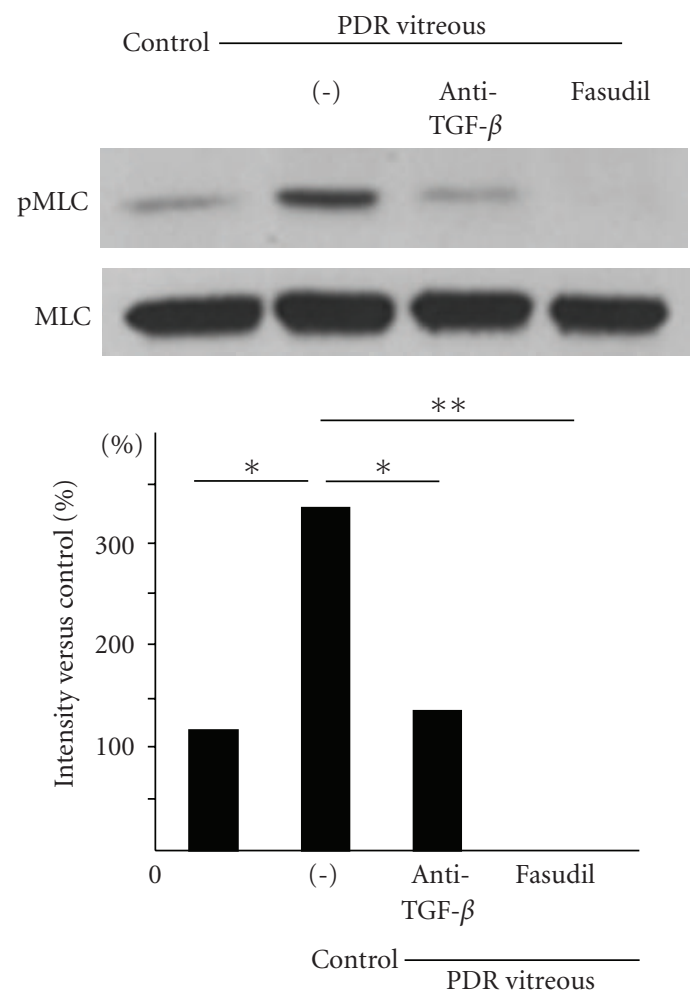

(b)

Figure 2: Continued. 


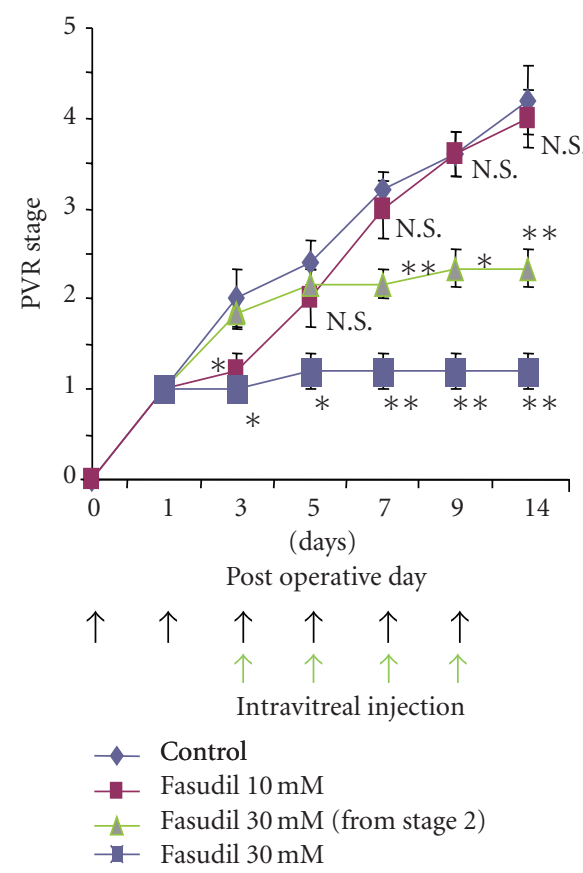

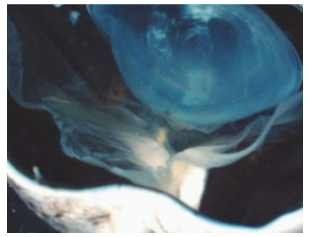

PVR (stage 5)

(d)

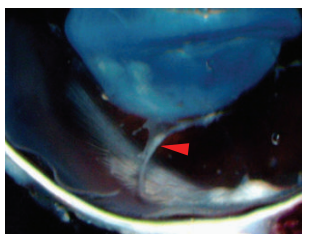

PVR + fasudil $30 \mathrm{mM}$ (stage 2)

(g)

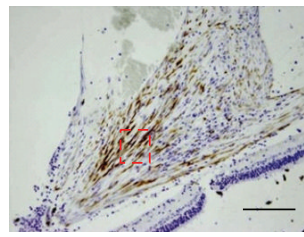

PVR (stage 5)

(e)

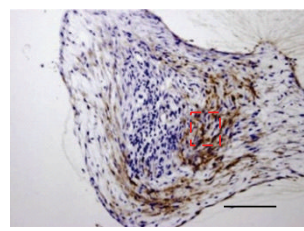

PVR + fasudil $30 \mathrm{mM}$ (stage 2)

(h)

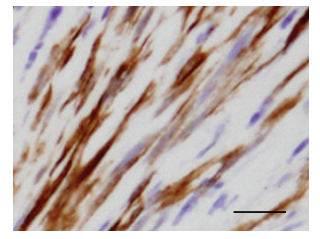

PVR (stage 5)

(f)

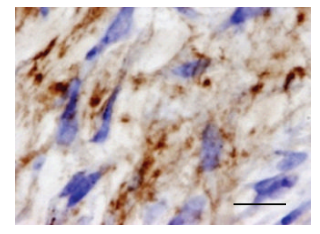

PVR + fasudil $30 \mathrm{mM}$ (stage 2)

(i)

(c)

FIGURE 2: Impact of fasudil on PDR vitreous-induced collagen gel contraction and MLC phosphorylation. After pretreatment with or without anti-TGF- $\beta$ mAb or fasudil, hyalocytes were stimulated with vitreous with PDR. (a) In hyalocyte-containing collagen gels, fasudil almost completely suppressed the contraction of collagen gels treated with PDR vitreous. The diameter of the gels was measured and statistically analyzed $\left({ }^{*} P<.05 ;{ }^{* *} P<.01\right.$; NS, not significant, $n=3$ each). (b) Western blot analysis was performed to detect phosphorylated MLC (pMLC). Fasudil abolished MLC phosphorylation, induced by PDR vitreous. Lane-loading differences were normalized by MLC. Signal intensities were quantified and expressed as percentages of the pMLC/MLC ratio compared with control $\left({ }^{*} P<.05 ;{ }^{*} P<.01, n=3\right.$ each). Experimental PVR in rabbit eyes. (c) Therapeutic potential of fasudil in reducing the progression of experimental PVR. PVR was classified into six stages $(0-5)$. Rhombus, vehicle $(n=5)$; purple square, fasudil $10 \mu \mathrm{M}(n=5)$; trigone, fasudil $30 \mu \mathrm{M}$ from stage $2(n=6)$; blue square, fasudil $30 \mu \mathrm{M}(n=5)\left({ }^{*} P<.05,{ }^{* *} P<.01\right.$, not significant versus vehicle). (d) Tractional retinal detachment because of formation and cicatricial contraction of preretinal proliferative membrane was observed by stereomicroscopy in vehicle-treated eyes (stage 5 PVR). (g) In contrast, intravitreal membranes adhered to the retina without causing retinal detachment (arrowhead) in $30 \mu \mathrm{M}$ fasudil-treated eyes with stage 2 PVR. Micrographs depict $\alpha$-SMA expression (brown) in preretinal proliferative membrane with stage 5 PVR (e) and stage 2 PVR (h) by immunohistochemical analysis. (Scale bar, $200 \mu \mathrm{m}$ ). (f and i) Magnified images of (e) and (h), respectively, (Scale bar, $10 \mu \mathrm{m})$.

as a new strategy in the total management of DR from its early to late stages.

\section{Involvement of Rho/ROCK Pathway in the Pathogenesis of Diabetic Retinopathy}

Recent studies have revealed that small GTP-binding protein Rho and its target protein ROCK are implicated in the important physiological roles such as cell adhesion and migration mediated through MLC phosphorylation $[19,24]$. Rho activity is also increased in bovine aortic endothelial cells treated with high glucose [25], and involved in the pathogenesis of renal and aortic complications during diabetic states [26, 27]. Furthermore, we revealed that Rho/ROCK pathway is activated in retinal microvessels during diabetes (Figures 1(a)-1(e)).
Rho/ROCK pathway promotes leukocyte adhesion to the microvasculature by affecting the expression and function of adhesion molecules, including ICAM-1 [28, 29] and integrins [30]. Moreover, ROCK causes firm adhesion through the activation of ezrin, radixin, and moesin in endothelial cells, which jointly form the anchoring structures for leukocytes' integrins [31, 32]. These findings suggest that elevated activity of the Rho/ROCK pathway is involved in the pathogenesis of diabetic microvasculopathy mediated through leukocyte adhesion.

Rho/ROCK signaling is also involved in the pathogenesis of VEGF-induced angiogenesis. In endothelial cells ROCK pathway plays a critical role in VEGF-induced endothelial migration by regulating stress fiber formation associated with MLC phosphorylation [17, 33]. Moreover, recent in vivo studies have also demonstrated that the ROCK pathway plays a critical role in angiogenesis $[34,35]$. 


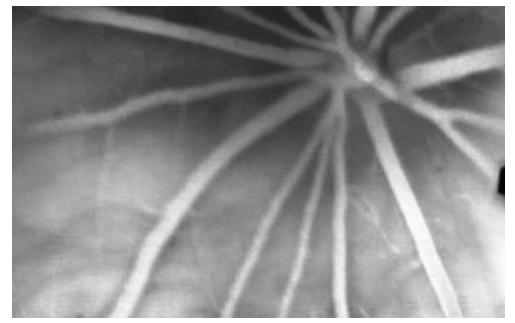

(a)

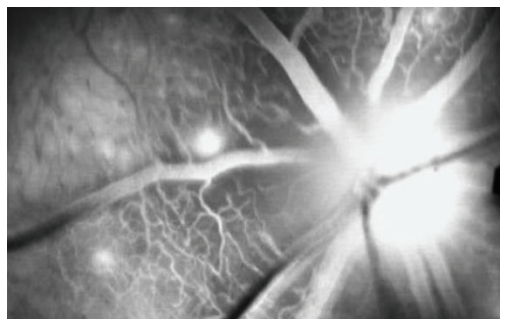

(b)

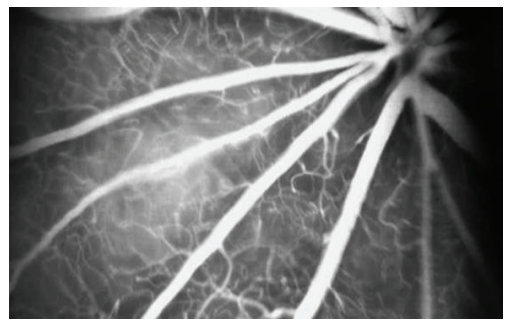

(c)

FIgURE 3: Contractile impacts of ROCK activation in rat retinal vasculature. Intravitreal injections ofllysophosphatidic acid (LPA), Rho activator, were performed into rat's eyes over a period of 1 minute with a 33-gauge needle. The final intraocular concentration of LPA was $20 \mu \mathrm{M}$. We monitored the retinal fluorescein with a scanning laser ophthalmoscope ((a) no injection, (b) 5 minutes after injection, (c) 10 minutes after injection). Intravitreal injection of induced sever retinal vessel contraction.

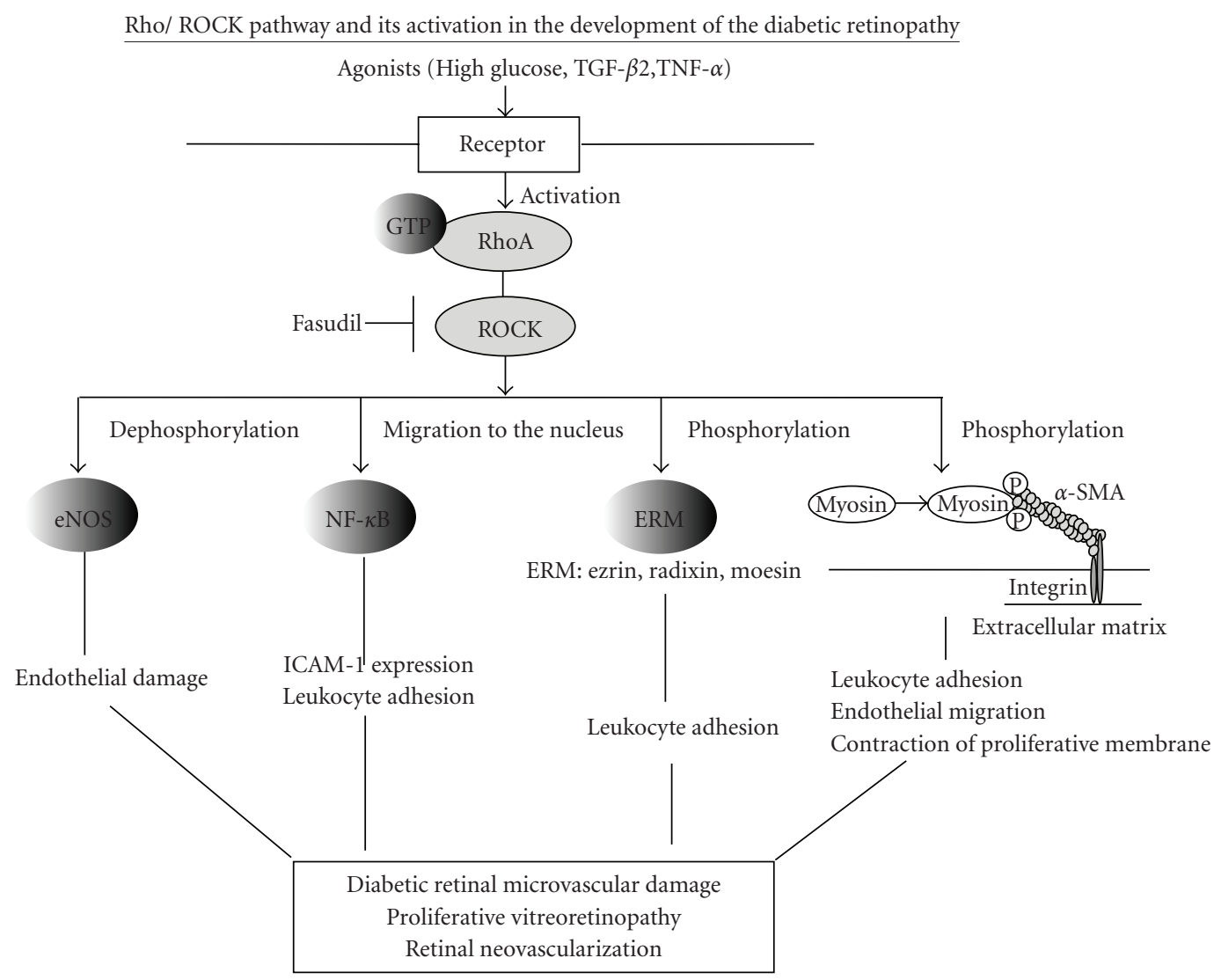

FIGURE 4: Rho/ROCK pathway and its activation in the development of diabetic retinopathy. RhoA is a small GTP-binding protein, and Rho-kinase (ROCK) is its target protein. Glucose, TNF- $\alpha$, and TGF- $\beta$, elevated in diabetic serum or vitreous, activate Rho/ROCK pathway in endothelial cells or hyalocytes. ROCK activation induces endothelial damage mediated through inactivation of endothelial nitric oxide synthase (eNOS), which has endothelial protective potential. Moreover, ROCK causes firm leukocyte adhesion through the increase of ICAM-1 expression and activation of ezrin, radixin, and moesin (ERM) in endothelial cells. ROCK also has important roles such as leukocyte adhesion, endothelial migration, and contraction of proliferative membrane mediated through myosin light chain (MLC) phosphorylation in diabetic retinopathy. These findings suggest that elevated activity of the Rho/ROCK pathway is involved in the pathogenesis of diabetic microvascular damage, proliferative vitreoretinopathy, and retinal neovascularization. 
Previously we showed TGF- $\beta 2$ contributes to transdifferentiation of hyalocytes into $\alpha$-SMA positive myofibroblastlike cells that causes hyalocyte-containing collagen gel contraction [19]. Moreover, we revealed that TGF- $\beta 2$, overexpressed in the vitreous and contractile membranes of PDR patients, activates ROCK pathway and forms stress fibers and contractions mediated through ROCK activation [20, 23]. These results suggest the central role of ROCK in the cicatricial contraction of proliferative membrane in PDR patients.

\section{Therapeutic Strategy in Diabetic Retinopathy by ROCK Inhibition}

3.1. ROCK Inhibition Ameliorates Diabetes-Induced Microvascular Damage. Fasudil, a potent and selective ROCK inhibitor, is relatively safe and effective in the treatment of cardiovascular disease including cerebral and coronary vasospasm, angina, hypertension, and heart failure with no serious adverse side effect in fasudil-treated patients [36]. In our animal experiments, intravitreal injection of fasudil did not cause apparent electrophysiological or morphological changes in retinal tissues at least within its effective concentrations [23]. However, several adverse effects such as hepatic function abnormal, intracranial hemorrhage, and hypotension have been reported [37, 38]. We thus need further examination regarding the safety and adverse effects of ROCK inhibitor before its clinical use in the field of intraocular diseases.

Nevertheless, we recently could reveal the therapeutic potential of fasudil in the management of earlier stages of DR. Treatment with intravitreal injection of fasudil significantly decreased retinal leukocyte adhesion in diabetic rat mediated through reduction of ROCK activation. Moreover, fasudil effectively suppressed endothelial damage, even when leukocytes firmly adhered to the endothelium (Figures 1(f)-1(i)). This suggests that fasudil directly causes endothelial protection in addition to its impact on leukocyte adhesion. Rho/ROCK inactivates endothelial nitric oxide synthase (eNOS) in human umbilical venous cells [39]. eNOS generates physiological levels of nitric oxide (NO), a potent vasodilator [40] and antiapoptotic factor [41, 42]. Fasudil treatment almost completely reversed the decreased eNOS activity in diabetic rat retinas. In addition, the protective effect of fasudil on microvascular endothelial cells was significantly blocked by NOS inhibition with L-NAME, without apparent effect on leukocyte adhesion in vitro. These findings suggest that fasudil has a direct endothelial protective potential through induction of physiological levels of NO, synthesized by eNOS.

\subsection{Antiangiogenic Properties of ROCK Inhibitor. We} demonstrated that ROCK inhibitor could inhibit VEGF-elicited bovine retinal capillary endothelial cell (BREC) migration and proliferation in vitro and corneal neovascularization in vivo. A ROCK inhibitor fasudil had inhibitory effect on BREC migration with a scratchwound assay. Moreover, fasudil could inhibit VEGFinduced BRECs $\left[{ }^{3} \mathrm{H}\right]$-thymidine incorporation and ERK1/2 phosphorylation, whose activity indicates the proliferative activities of endothelial cells in angiogenic processes [15]. In vivo, fasudil strongly attenuated VEGF-induced corneal neovascularization in a corneal pocket assay [43].

3.3. ROCK Inhibition Suppresses Critical Contraction of Proliferative Membrane. We could also demonstrate the therapeutic potential of ROCK inhibitor fasudil in the management of later stages of DR. In hyalocyte-containing collagen gels assay, fasudil almost completely abolished the PDR vitreous-induced collagen gel contraction mediated through the suppression of MLC phosphorylation (Figures 2(a) and 2(b)). In experimental PVR rabbit model, fasudil also effectively disrupted $\alpha$-SMA organization and blocked contraction of proliferative membrane (Figures 2(c)-2(i)).

Statins, inhibitors of the 3-hydroxy-3-methyl-glutaryl(HMG-) CoA reductase, are widely used to reduce endogenous cholesterol synthesis and improve hypercholesterolemia [44]. By inhibiting HMG-CoA reductase, statins also block ROCK activation mediated through the mevalonate pathway [45].

We demonstrated that simvastatin almost completely inhibited vitreous-induced contraction of the collagen gels in exvivo and proliferative membrane in experimental PVR model mediated through ROCK inhibition [46]. Our results indicate that ROCK inhibition suppresses PVR progression in later DR stages.

\subsection{Other Beneficial Effects of Fasudil on \\ Retinal Tissue during Diabetes}

3.4.1. Vasodilatory Property and Improvement of Hemodynamics in the Retinal Vessels. Development of chronic retinal ischemic state aggravates diabetic retinopathy. Rho/ROCK activation plays an important role in the pathogenesis of vasoconstriction, such as cerebral and coronary spasm [47] or hypertension [48], by NO-dependent mechanisms. We could show that intravitreal injection of lysophosphatidic acid, a potential Rho activator, induced severe retinal vessel constriction (Figures 3(a)-3(c)). Recent studies suggested that ROCK inhibitor fasudil improved hemodynamic states in human [49], and also dilated rat retinal vessels, and increases blood flow [48]. These results suggest that fasudil has preventable benefit on retinal ischemia during diabetes through improvement of hemodynamics in the retinal vessels.

3.4.2. Retinal Neuroprotective Effect of Fasudil. Retinal ischemia secondary to DR causes functional and irreversible damage not only in retina vasculature but also in retinal neuronal cells. Chronic loss of neuronal cells from the inner retina by increasing the frequency of apoptosis reduces the thickness of the nerve fiber layer in diabetic retina [50]. Impaired retinal electrophysiology and neurodegeneration have been shown in diabetic patients $[51,52]$. Recent studies revealed that Rho/ROCK pathway also seems to be associated with the pathogenesis of this neuronal damage. Abnormal activation of the Rho/ROCK pathway is important in the 
pathogenesis of several neurological diseases [53]. In rat retina, Rho/ROCK pathway is also involved in N-methyl-Daspartat-induced neurotoxicity in the rat retina. [54]. These studies suggest that ROCK inhibitor would protect against neuronal damage by acting directly on neurons. In fact, the ROCK inhibitor Y-27632 increases regeneration of retinal ganglion cell in the rat optic nerve crush model [55]. Moreover, ROCK inhibition attenuates ischemia-induced retinal neuronal cell death by inhibiting leukocytes extravasation and release of proinflammatory cytokines such as TNF- $\alpha$ or IL-6 in vitro/vivo [56, 57]. These data suggest that inhibition of Rho/ROCK pathway leads to neuroprotective effect and promote retinal cell survival during diabetes.

\section{Conclusion}

Rho/ROCK pathway is involved throughout the pathogenesis of DR, particularly in diabetic retinal microvasculopathy, neovascularization, and tractional retinal detachment associated with cicatricial contraction of preretinal proliferative membranes (Figure 4). Since we must consider frequent intravitreal injections as administration method due to a short biological half-life time of the compound in the vitreous cavity, we are considering intravitreal implantation of a slowly releasing drug-delivery system. For preventable benefit on progressing retinal microvascular damage and keeping good visual acuity, timing of intravitreal fasudil implantation prefers when early clinical DR findings such as microaneurysm begin to appear. In addition, pre- and postoperative intravitreal implantations for active PDR patients with proliferative membrane are also considered to be effective for prevention of PVR and tractional retinal detachment. Whereas further basic and clinical studies to reveal the effectiveness and safety of ROCK inhibitor are needed for clinical use in the field of eye diseases, ROCK inhibition might become a novel therapeutic strategy in the total management of DR from its early to late stages.

\section{References}

[1] J. H. Kempen, B. J. O'Colmain, M. C. Leske, et al., "The prevalence of diabetic retinopathy among adults in the United States," Archives of Ophthalmology, vol. 122, no. 4, pp. 552563, 2004.

[2] H. King, R. E. Aubert, and W. H. Herman, "Global burden of diabetes, 1995-2025: prevalence, numerical estimates, and projections," Diabetes Care, vol. 21, no. 9, pp. 1414-1431, 1998.

[3] "Early photocoagulation for diabetic retinopathy. ETDRS report number 9. Early Treatment Diabetic Retinopathy Study Research Group," Ophthalmology, vol. 98, pp. 766-785, 1991.

[4] L. P. Aiello, "Angiogenic pathways in diabetic retinopathy," The New England Journal of Medicine, vol. 353, no. 8, pp. 839-841, 2005.

[5] D. S. Fong, L. Aiello, T. W. Gardner, et al., "Diabetic retinopathy," Diabetes Care, vol. 26, no. 1, pp. S99-S102, 2003.

[6] A. M. Joussen, T. Murata, A. Tsujikawa, B. Kirchhof, S.-E. Bursell, and A. P. Adamis, "Leukocyte-mediated endothelial cell injury and death in the diabetic retina," American Journal of Pathology, vol. 158, no. 1, pp. 147-152, 2001.
[7] C. W. Smith, S. D. Marlin, R. Rothlein, C. Toman, and D. C. Anderson, "Cooperative interactions of LFA-1 and Mac-1 with intracellular adhesion molecule-1 in facilitating adherence and transendothelial migration of human neutrophils in vitro," Journal of Clinical Investigation, vol. 83, no. 6, pp. 20082017, 1989.

[8] F. C. Barouch, K. Miyamoto, J. R. Allport, et al., "Integrinmediated neutrophil adhesion and retinal leukostasis in diabetes," Investigative Ophthalmology and Visual Science, vol. 41, no. 5, pp. 1153-1158, 2000.

[9] A. M. Joussen, V. Poulaki, N. Mitsiades, et al., "Suppression of Fas-FasL-induced endothelial cell apoptosis prevents diabetic blood-retinal barrier breakdown in a model of streptozotocininduced diabetes," The FASEB Journal, vol. 17, no. 1, pp. 7678, 2003.

[10] R. Arita, Y. Hata, S. Nakao, et al., "Rho kinase inhibition by fasudil ameliorates diabetes-induced microvascular damage," Diabetes, vol. 58, no. 1, pp. 215-226, 2009.

[11] N. Ferrara, "Vascular endothelial growth factor: basic science and clinical progress," Endocrine Reviews, vol. 25, no. 4, pp. 581-611, 2004.

[12] A. P. Adamis and D. T. Shima, "The role of vascular endothelial growth factor in ocular health and disease," Retina, vol. 25, no. 2, pp. 111-118, 2005.

[13] S. Moradian, H. Ahmadieh, M. Malihi, M. Soheilian, M. H. Dehghan, and M. Azarmina, "Intravitreal bevacizumab in active progressive proliferative diabetic retinopathy," Graefe's Archive for Clinical and Experimental Ophthalmology, vol. 246, no. 12, pp. 1699-1705, 2008.

[14] C. Shima, H. Sakaguchi, F. Gomi, et al., "Complications in patients after intravitreal injection of bevacizumab," Acta Ophthalmologica, vol. 86, no. 4, pp. 372-376, 2008.

[15] B. P. Eliceiri, R. Klemke, S. Stromblad, and D. A. Cheresh, "Integrin $\alpha \mathrm{v} \beta 3$ requirement for sustained mitogen-activated protein kinase activity during angiogenesis," Journal of Cell Biology, vol. 140, no. 5, pp. 1255-1263, 1998.

[16] G. Mavria, Y. Vercoulen, M. Yeo, et al., "ERK-MAPK signaling opposes Rho-kinase to promote endothelial cell survival and sprouting during angiogenesis," Cancer Cell, vol. 9, no. 1, pp. 33-44, 2006.

[17] G. P. van Nieuw Amerongen, P. Koolwijk, A. Versteilen, and V. W. M. Van Hinsbergh, "Involvement of RhoA/Rho kinase signaling in VEGF-induced endothelial cell migration and angiogenesis in vitro," Arteriosclerosis, Thrombosis, and Vascular Biology, vol. 23, no. 2, pp. 211-217, 2003.

[18] Y. Noda, Y. Hata, T. Hisatomi, et al., "Functional properties of hyalocytes under PDGF-rich conditions," Investigative Ophthalmology and Visual Science, vol. 45, no. 7, pp. 21072114, 2004.

[19] K. Hirayama, Y. Hata, Y. Noda, et al., "The involvement of the Rho-kinase pathway and its regulation in cytokineinduced collagen gel contraction by hyalocytes," Investigative Ophthalmology and Visual Science, vol. 45, no. 11, pp. 38963903, 2004.

[20] T. Kita, Y. Hata, K. Kano, et al., "Transforming growth factor- $\beta 2$ and connective tissue growth factor in proliferative vitreoretinal diseases: possible involvement of hyalocytes and therapeutic potential of rho kinase inhibitor," Diabetes, vol. 56, no. 1, pp. 231-238, 2007.

[21] K. E. Kamm and J. T. Stull, "The function of myosin and myosin light chain kinase phosphorylation in smooth muscle," Annual Review of Pharmacology and Toxicology, vol. 25, pp. 593-620, 1985. 
[22] Y. Fukata, K. Kaibuchi, M. Amano, and K. Kaibuchi, "RhoRho-kinase pathway in smooth muscle contraction and cytoskeletal reorganization of non-muscle cells," Trends in Pharmacological Sciences, vol. 22, no. 1, pp. 32-39, 2001.

[23] T. Kita, Y. Hata, R. Arita, et al., "Role of TGF- $\beta$ in proliferative vitreoretinal diseases and ROCK as a therapeutic target," Proceedings of the National Academy of Sciences of the United States of America, vol. 105, no. 45, pp. 17504-17509, 2008.

[24] K. Kaibuchi, S. Kuroda, and M. Amano, "Regulation of the cytoskeleton and cell adhesion by the Rho family GTPases in mammalian cells," Annual Review of Biochemistry, vol. 68, pp. 459-486, 1999.

[25] H. Iwasaki, R. Okamoto, S. Kato, et al., "High glucose induces plasminogen activator inhibitor-1 expression through Rho/Rho-kinase-mediated NF- $\kappa \mathrm{B}$ activation in bovine aortic endothelial cells," Atherosclerosis, vol. 196, no. 1, pp. 22-28, 2008.

[26] V. Kolavennu, L. Zeng, H. Peng, Y. Wang, and F. R. Danesh, "Targeting of RhoA/ROCK signaling ameliorates progression of diabetic nephropathy independent of glucose control," Diabetes, vol. 57, no. 3, pp. 714-723, 2008.

[27] J. Tang, I. Kusaka, A. R. Massey, S. Rollins, and J. H. Zhang, "Increased RhoA translocation in aorta of diabetic rats," Acta Pharmacologica Sinica, vol. 27, no. 5, pp. 543-548, 2006.

[28] H. Lee, I. L. Chi, J.-J. Liao, et al., "Lysophospholipids increase ICAM-1 expression in HUVEC through a G i- and NF- $\kappa \mathrm{B}-$ dependent mechanism," American Journal of Physiology, vol. 287, no. 6, pp. C1657-C1666, 2004.

[29] B. Wojciak-Stothard, L. Williams, and A. J. Ridley, "Monocyte adhesion and spreading on human endothelial cells is dependent on Rho-regulated receptor clustering," Journal of Cell Biology, vol. 145, no. 6, pp. 1293-1307, 1999.

[30] C. Giagulli, E. Scarpini, L. Ottoboni, et al., "RhoA and $\zeta$ PKC control distinct modalities of LFA-1 activation by chemokines: critical role of LFA-1 affinity triggering in lymphocyte in vivo homing," Immunity, vol. 20, no. 1, pp. 25-35, 2004.

[31] O. Barreiro, M. Yanez-Mo, J. M. Serrador, et al., "Dynamic interaction of VCAM-1 and ICAM-1 with moesin and ezrin in a novel endothelial docking structure for adherent leukocytes," Journal of Cell Biology, vol. 157, no. 7, pp. 1233-1245, 2002.

[32] D. J. G. Mackay, F. Esch, H. Furthmayr, and A. Hall, "Rhoand Rac-dependent assembly of focal adhesion complexes and actin filaments in permeabilized fibroblasts: an essential role for ezrin/radixin/moesin proteins," Journal of Cell Biology, vol. 138, no. 4, pp. 927-938, 1997.

[33] L. Zeng, H. Xu, T.-L. Chew, et al., "HMG CoA reductase inhibition modulates VEGF-induced endothelial cell hyperpermeability by preventing RhoA activation and myosin regulatory light chain phosphorylation," FASEB Journal, vol. 19, no. 13, pp. 1845-1847, 2005.

[34] M. V. Hoang, M. C. Whelan, and D. R. Senger, "Rho activity critically and selectively regulates endothelial cell organization during angiogenesis," Proceedings of the National Academy of Sciences of the United States of America, vol. 101, no. 7, pp. 1874-1879, 2004.

[35] J.-M. Hyvelin, K. Howell, A. Nichol, C. M. Costello, R. J. Preston, and P. McLoughlin, "Inhibition of Rho-kinase attenuates hypoxia-induced angiogenesis in the pulmonary circulation," Circulation Research, vol. 97, no. 2, pp. 185-191, 2005.

[36] H. Shimokawa and M. Rashid, "Development of Rho-kinase inhibitors for cardiovascular medicine," Trends in Pharmacological Sciences, vol. 28, no. 6, pp. 296-302, 2007.
[37] M. Shibuya, Y. Suzuki, K. Sugita, et al., "Dose escalation trial of a novel calcium antagonist, AT877, in patients with aneurysmal subarachnoid haemorrhage," Acta Neurochirurgica, vol. 107, no. 1-2, pp. 11-15, 1990.

[38] M. Shibuya, S. Hirai, M. Seto, S.-I. Satoh, and E. Ohtomo, "Effects of fasudil in acute ischemic stroke: results of a prospective placebo-controlled double-blind trial," Journal of the Neurological Sciences, vol. 238, no. 1-2, pp. 31-39, 2005.

[39] X.-F. Ming, H. Viswambharan, C. Barandier, et al., "Rho GTPase/Rho kinase negatively regulates endothelial nitric oxide synthase phosphorylation through the inhibition of protein kinase B/Akt in human endothelial cells," Molecular and Cellular Biology, vol. 22, no. 24, pp. 8467-8477, 2002.

[40] J. Marin and M. A. Rodriguez-Martinez, "Role of vascular nitric oxide in physiological and pathological conditions," Pharmacology and Therapeutics, vol. 75, no. 2, pp. 111-134, 1997.

[41] L. Rossig, B. Fichtlscherer, K. Breitschopf, et al., "Nitric oxide inhibits caspase-3 by S-nitrosation in vivo," The Journal of Biological Chemistry, vol. 274, no. 11, pp. 6823-6826, 1999.

[42] L. Rossig, J. Haendeler, C. Hermann, et al., "Nitric oxide down-regulates MKP-3 mRNA levels: involvement in endothelial cell protection from apoptosis," The Journal of Biological Chemistry, vol. 275, no. 33, pp. 25502-25507, 2000.

[43] Y. Hata, M. Miura, S. Nakao, S. Kawahara, T. Kita, and T. Ishibashi, "Antiangiogenic properties of fasudil, a potent Rhokinase inhibitor," Japanese Journal of Ophthalmology, vol. 52, no. 1, pp. 16-23, 2008.

[44] J. Molgaard, H. von Schenck, and A. G. Olsson, "Effects of simvastatin on plasma lipid, lipoprotein and apolipoprotein concentrations in hypercholesterolaemia," European Heart Journal, vol. 9, no. 5, pp. 541-551, 1988.

[45] L. Van Aelst and C. D'Souza-Schorey, "Rho GTPases and signaling networks," Genes and Development, vol. 11, no. 18, pp. 2295-2322, 1997.

[46] S. Kawahara, Y. Hata, T. Kita, et al., "Potent inhibition of cicatricial contraction in proliferative vitreoretinal diseases by statins," Diabetes, vol. 57, no. 10, pp. 2784-2793, 2008.

[47] A. Masumoto, M. Mohri, H. Shimokawa, L. Urakami, M. Usui, and A. Takeshita, "Suppression of coronary artery spasm by the Rho-kinase inhibitor fasudil in patients with vasospastic angina," Circulation, vol. 105, no. 13, pp. 1545-1547, 2002.

[48] N. Okamura, M. Saito, A. Mori, et al., "Vasodilator effects of fasudil, a Rho-kinase inhibitor, on retinal arterioles in strokeprone spontaneously hypertensive rats," Journal of Ocular Pharmacology and Therapeutics, vol. 23, no. 3, pp. 207-212, 2007.

[49] A. Masumoto, Y. Hirooka, H. Shimokawa, K. Hironaga, S. Setoguchi, and A. Takeshita, "Possible involvement of Rhokinase in the pathogenesis of hypertension in humans," Hypertension, vol. 38, no. 6, pp. 1307-1310, 2001.

[50] A. J. Barber, "A new view of diabetic retinopathy: a neurodegenerative disease of the eye," Progress in NeuroPsychopharmacology and Biological Psychiatry, vol. 27, no. 2, pp. 283-290, 2003.

[51] V. Parisi and L. Uccioli, "Visual electrophysiological responses in persons with type 1 diabetes," Diabetes/Metabolism Research and Reviews, vol. 17, no. 1, pp. 12-18, 2001.

[52] E. Lieth, T. W. Gardner, A. J. Barber, and D. A. Antonetti, "Retinal neurodegeneration: early pathology in diabetes," Clinical and Experimental Ophthalmology, vol. 28, no. 1, pp. 3-8, 2000. 
[53] B. K. Mueller, H. Mack, and N. Teusch, "Rho kinase, a promising drug target for neurological disorders," Nature Reviews Drug Discovery, vol. 4, no. 5, pp. 387-398, 2005.

[54] Y. Kitaoka, Y. Kitaoka, T. Kumai, et al., "Involvement of RhoA and possible neuroprotective effect of fasudil, a Rho kinase inhibitor, in NMDA-induced neurotoxicity in the rat retina," Brain Research, vol. 1018, no. 1, pp. 111-118, 2004.

[55] P. Lingor, N. Teusch, K. Schwarz, et al., "Inhibition of Rho kinase (ROCK) increases neurite outgrowth on chondroitin sulphate proteoglycan in vitro and axonal regeneration in the adult optic nerve in vivo," Journal of Neurochemistry, vol. 103, no. 1, pp. 181-189, 2007.

[56] A. Hirata, M. Inatani, Y. Inomata, et al., "Y-27632, a Rhoassociated protein kinase inhibitor, attenuates neuronal cell death after transient retinal ischemia," Graefe's Archive for Clinical and Experimental Ophthalmology, vol. 246, no. 1, pp. 51-59, 2008.

[57] A. Tura, F. Schuettauf, P. P. Monnier, K. U. Bartz-Schmidt, and S. Henke-Fahle, "Efficacy of Rho-kinase inhibition in promoting cell survival and reducing reactive gliosis in the rodent retina," Investigative Ophthalmology \& Visual Science, vol. 50, no. 1, pp. 452-461, 2009. 


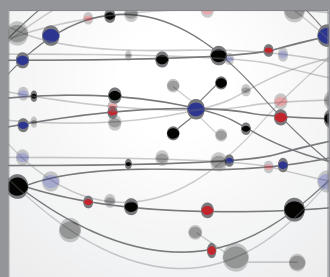

The Scientific World Journal
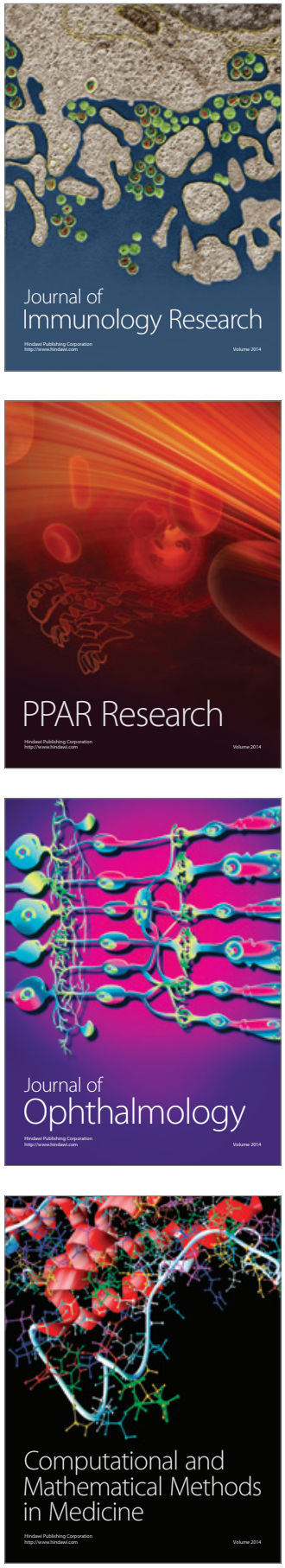

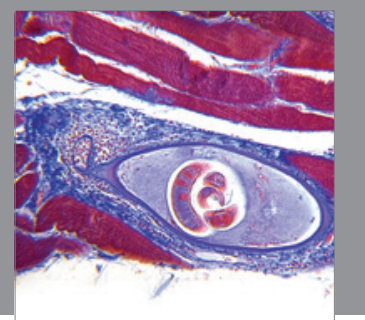

Gastroenterology

Research and Practice
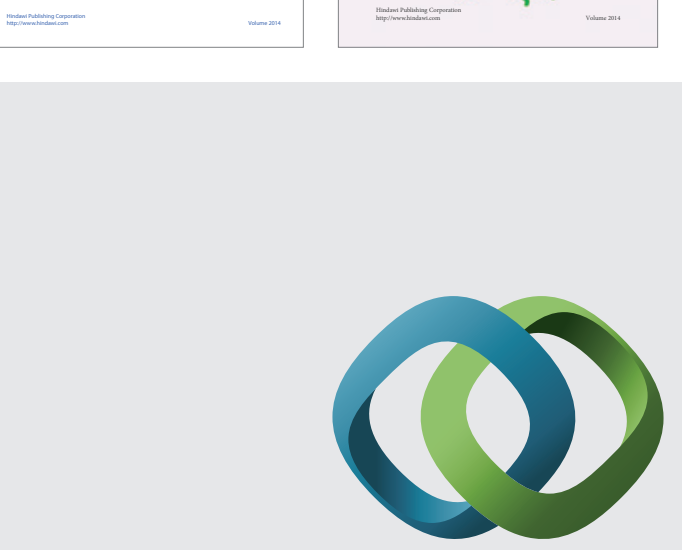

\section{Hindawi}

Submit your manuscripts at

http://www.hindawi.com
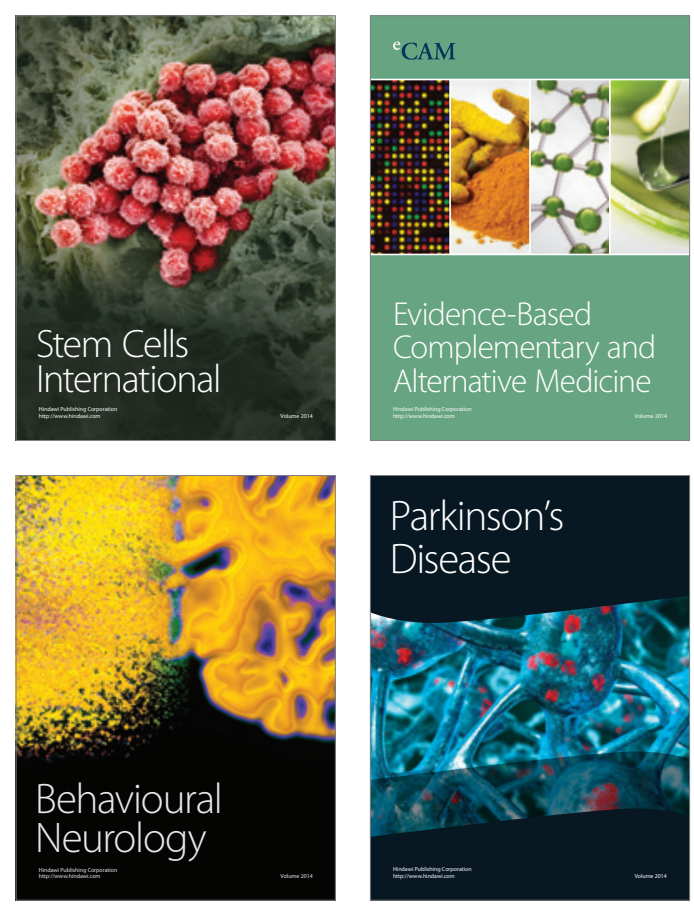

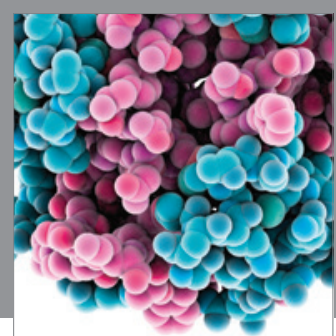

Journal of
Diabetes Research

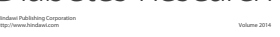

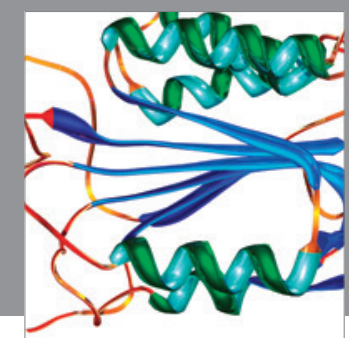

Disease Markers
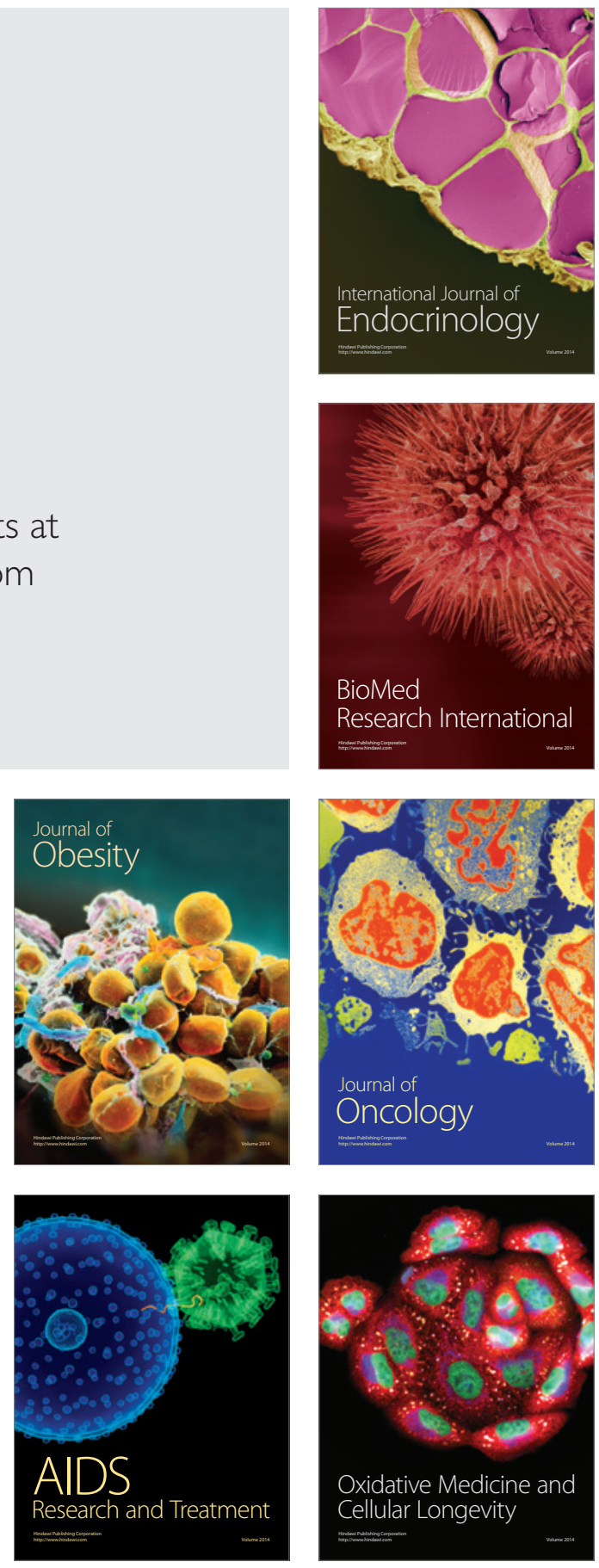